\title{
Bring CLARITY to Temporal Lobe Epilepsy: 3D Visualization of p-Tau(Ser262) and 14-3-3 Zeta
}

\author{
Qingqin Wu, Honghong Song, Juan Feng*, Yang Xia, Dezhong Yao \\ College of Life Science and Technology, University of Electronic Science and Technology of China, Chengdu, China
}

Email address:

fengjuan@uestc.edu.cn (Juan Feng)

${ }^{*}$ Corresponding author

\section{To cite this article:}

Qingqin Wu, Honghong Song, Juan Feng, Yang Xia, Dezhong Yao. Bring CLARITY to Temporal Lobe Epilepsy: 3D Visualization of p-Tau(Ser262) and 14-3-3 Zeta. International Journal of Biomedical Science and Engineering. Vol. 5, No. 6, 2017, pp. 63-67. doi: $10.11648 /$ j.ijbse.20170506.11

Received: July 30, 2017; Accepted: September 25, 2017; Published: December 6, 2017

\begin{abstract}
CLARITY is one new technology which allows the brain tissue become transparent. It has successfully been combined with immunofluorescence staining to achieve the 3D visualization of some molecules or neuronal cells in some disease brains. The temporal lobe epilepsy (TLE) is one neuronal disease which is characterized by the sprouting of the massy fibers (MSF). Previous study has showed that MSF could be affected by the phosphorylation at site-262 of microtubule association protein Tau (p-tau (Ser262)). However, in TLE little useful information was reported concerning the 3D architecture of p-tau (Ser262) and its relationship with 14-3-3 zeta that regulated the phosphorylation of Tau in AD disease. In this paper, pilocarpine-induced epilepsy model was established and identified by Timm-staining. Double immunofluorescent staining results showed that the development of TLE gave rise to the colocalization of p-tau (Ser262) and 14-3-3 zeta protein in CA1 and CA3 zone in hippocampi. The mm-thick brain sections were passively clarified, and 3D reconstruction imaging of the immunofluorescent staining showed that p-tau (Ser262) was diverse cluster-like shape. These results proved that CLARITY could be used to study TLE, in which the 3D morphologic changes of p-tau (Ser262) and the role of 14-3-3 zeta in the regulation of Tau needed to be further investigated.
\end{abstract}

Keywords: Temporal Lobe Epilepsy, CLARITY, Immunofluorescence, The Phosphorylation of Tau, 14-3-3 Zeta Protein

\section{Introduction}

CLARITY (Clear Lipid-exchanged Acrylamidehybridized Rigid Imaging / Immunostaining / in situhybridization-compatible Tissue hydrogel) is one new technology which allows the brain tissue become transparent [1]. Different from those optically-clearing reagents (including BABB [2], 3DISCO [3], Sca/e [4], See DB [5], Cubic [6]), which minimize the light scattering by changing the refractive index of the thick tissues, SDS detergent is used in CLARITY to remove the lipids rich in the brains. Most proteins and nucleotides are retained in the brain by being covalently linked to the nanoporous hydrogel matrix formed prior to SDS-treatment. Following the clearing step, the clarified tissue is immersed into the refraction index matching media (for example $85 \%$ glycerol) to make tissues more uniform and homogeneous. When compared with other clearing methods, CLARITY has the following advantages:
(1) The removing of the lipids allows the incident light to penetrate deeper tissue to achieve the mm-thick tissue imaging. (2) The macromolecular antibodies have more accesses to diffuse into the brain tissues containing the porous hydrogel. Meanwhile, the fluorescence coming from the antibodies labeled by fluorescence probes will not be easily quenched in CLARITY. These advantages have attracted more and more attention from the researchers worldwide. Some researchers have optimized the CLARITY conditions [7], and some have successfully extended CLARITY to other tissues (including pancreas, liver, kidney, lung, intestine and even plant leaf) and other fields [8, 9, 10, $11,12,13,14,15]$. More interestingly, in the recent years CLARIY has been successfully combined with immunofluorescent staining methods to achieve three-dimensional visualization of some cells or molecules in mm-thick tissues or even intact brain. For instance, Spence RD. et al investigated the relationship between gray 
matter atrophy and axonal injury by combing CLARITY and fNMR [16]. Ando K. et al uncovered the 3D architecture of $\mathrm{Ab}$, Tau and neurofibrillary tangles (NFT) in the $500 \mu \mathrm{m}$ clarified transparent frontal cortex of human post-mortem suffering from AD by combining CLARITY and confocal imaging [17]. Gentleman SM et al. visualized a characteristic spherical or near-spherical $\alpha$-synuclein $(\alpha \mathrm{SN})$ shell on the surface of the Lewy body inclusions in the clarified post-mortem Parkinson's brain. They also observed the the spatial relationship between monoaminergic fibres and Lewpathologies using anti-TH and anti-IBI1 as the antibodies, respectively [18]. Obviously, CLARITY has made some contributed to gain deeper insight on the morphological and pathological studies of some neuronal diseases.

Temporal lobe epilepsy (TLE) is one common neuronal disease. It is mainly characterized by the sprouting of mossy fiber (MFS), where $\mathrm{Zn}^{2+}$ is rich and it can be detected via Timm-staining reagents. At the beginning, Biliverdin was used as the probe for $\mathrm{Zn}^{2+}$ rich in MFS, but it failed in this work. Next, one microtube-associated protein tau was selected in this paper because its phosphorylation level at site 262 (donted as p-tau (Ser262)) could affect the degree of MFS. In other words, p-tau (Ser262) might be one promising target for epilepsy therapy. Numerous studies have showed that p-tau (Ser262) could be regulated by various kinases. Additionally, 14-3-3 zeta protein was also found to have capability to regulate the the phosphorylation level of Tau [19]. In AD, 14-3-3 zeta protein even affected the

The morphology of Tau varying from amorphous aggregation, single-stranded filaments, ribbon like filaments to the paired helical filaments (PHFs) [20]. Because little information has been available concerning the $3 \mathrm{D}$ architecture of p-tau (Ser262) and its relationship with 14-3-3 zeta protein in TLE, this paper aimed to explore these questions by combining CALRITY and immunofluorescent staining method.

In the present work the pilocarpine-induced temporal lobe epilepsy model was established on mice, and this model was identified via Timm-staining methods. The mm-thick brain slabs were clarified using passive diffusion method. The antibodies against p-tau (Ser262) and against 14-3-3 zeta protein were used for the double immunofluorescence staining. The distribution and 3D artechecture of both p-tau (Ser262) and 14-3-3 zeta protein were observed, and their spatial relationship was also detected. This result will help us to understand the in vivo role of 14-3-3 zeta in the regulation of p-Tau in TLE.

\section{Main Body}

\subsection{Antibodies and Chemicals}

$\mathrm{CsCl}$, Bromo-methyl scopolamine, pilocarpine and diazepam were purchased from Sigma company. Other reagents were bought from Kelong Chemical plant or the following company.
Table 1. List of reagents and antibodies used in the hydrogel solution and the immunofluorescent staining.

\begin{tabular}{lll}
\hline Reagent & Dilution & Source \\
\hline Acrylamide & & Biosharp \\
Bisacrylamide & Biosharp \\
VA-044 initiator & Sigma-Aldrich \\
Goat anti-human 14-3-3 zeta antibody & $1: 120$ & R\&D systems \\
Rabbit anti-mouse P-262 antibody & $1: 120$ & Abcam \\
Alexa 488-conjugated donkey anti-sheep IgG & $1: 150$ & Invitrogen \\
Alexa 569-conjugated donkey anti-sheep IgG & $1: 150$ & Invitrogen \\
DAPI & $1: 10000$ & Roche Group \\
Normal donkey serum & $1: 200$ & Solarbio \\
\hline
\end{tabular}

\subsection{Model of Epilepsies Mice, Behavior and Timm-Staining}

The 6-8 week-old Kunming mice were pretreated by $\mathrm{CsCl}$ solution and $1 \mathrm{mg} / \mathrm{mL}$ Bromo-methyl scopolamine. Following this pre-treatment, $\sim 230 \mathrm{mg} / \mathrm{mL}$ pilocarpine was administered to produce the status epileptics according to the reference [21]. Timm-staining experiment was done to test if the TLE model was successful or not. The double immunofluorescent staining was performed on $40 \mu \mathrm{m}$-thick mice brain section using anti-Tau (P-262) and anti-14-3-3 zeta antibodies.

\subsection{CLARITY of Mice Brain and MM-Thick Tissue}

According to the protocol described in the reference [7], CLARITY was performed on the mm-thick mice brain. In the first step, the elispy mice and the control group were deeply anesthetized using $10 \%$ hydrated chloral hydrate, and then these mice were transcardially perfused with $20 \mathrm{~mL}$ of ice cold $1 \times$ PBS followed by $20 \mathrm{~mL}$ of ice cold hydrogel PBS buffer containing (4\% acrylamide, $4 \%$ paraformaldehyde, $0.05 \%$ Bisacrylamide and $0.25 \%$ VA044 initiator). Subsequently, the brain tissue was extracted immediately and immersed in the hydrogel buffer at $4^{\circ} \mathrm{C}$ for 3 days. In the second step, after oxygen was removed by $\mathrm{N}_{2}$ bubbering, the hydrogel was polymerized by heating the solution at $37^{\circ} \mathrm{C}$ for 3 hours. Following this step, the mm-thick brain slices were obtained using Stainless Steel Mouse brain Slicer. In the third step, the brain slabs were immersed into $200 \mathrm{mM}$ boric acid clearing buffer ( $\mathrm{pH} 8.5$ ) containing 4\% SDS. During this step, the clearing solution was used each $48 \mathrm{~h}$ until the tissue had completely been clarified within one week.

\subsection{Immunostaining and Imaging of the Clarified mm-Thick Brain Slice}

The clarified slices were submerged in $1 \times \mathrm{PBS}$ buffer containing $0.1 \%$ Triton $\mathrm{X}-100$ at $37^{\circ} \mathrm{C}$ for at least $2 \mathrm{~d}$ so as to remove the clearing solution. After the slices were transferred into 48-well plates, the Goat-anti human 14-3-3 zeta primary antibody was introduced with 1:100 dilution, and the incubation was kept for 3 days. These slices were washed in PBST at room temperature for 1 day. Subsequently, the Alexa Fluor 488 Donkey-anti Goat secondary antibody was added at 1:150 into PBST. After 2-day incubation, the slices were washed in PBST three times for $30 \mathrm{~min}$. Dapi was also added and was kept overnight to stain the cellular nucleus. Prior to the imaging, the slices were then put into $85 \%$ glycerol at least 
$2 \mathrm{~h}$ for refractive index matching. Images were collected using Olympus BX51 confocal fluorescence microscopy. The 3D reconstructing was conducted using Imaris v7.4.2. software.

\section{Details}

In this paper, pilocarpine was administrated to induce the SE of mice. Different from the reference [20], the repeated administration of pilocarpine was used here instead of the single high-dose administration. In addition, the dose of pilocarpine was also optimized to $230 \mathrm{mg} / \mathrm{mL}$ to reduce the mortality rate of mice $(\sim 25 \%)$. As seen in Figure 1 , the repeated administration of pilocarpineat 20-min intervals result in the production of SE. Figure 2A-D gave the Timm-staining results of the control group and the treated group. Comparisons showed that the fiber sprouting was clearly detected in the pilocarpine-treated group (seen in Figure 2C), indicating that TLE had been successfully modeling on mice.

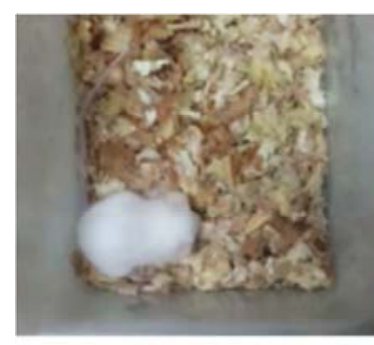

(a)

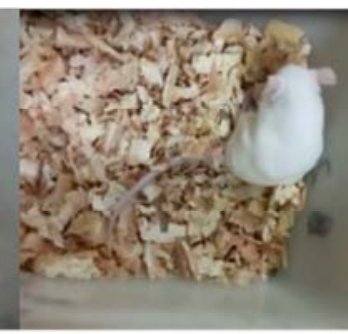

(b)

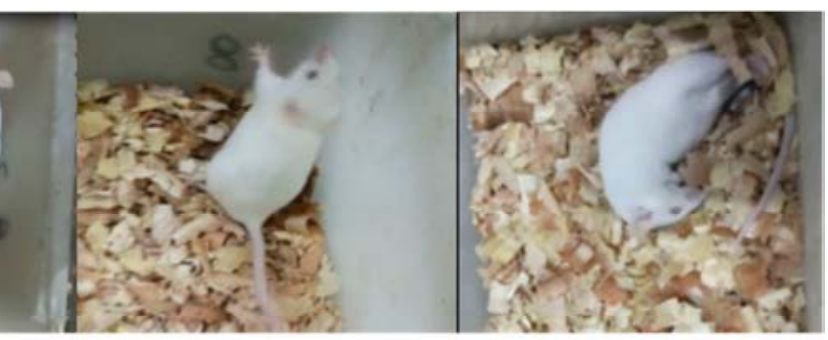

(c) (d)

Figure 1. The characteristic symptoms including rhythmic nodding, foreleg clonicity, hind leg standing-up, and falling down, representing grade II, grade III, grade $I V$ and grade $V$.

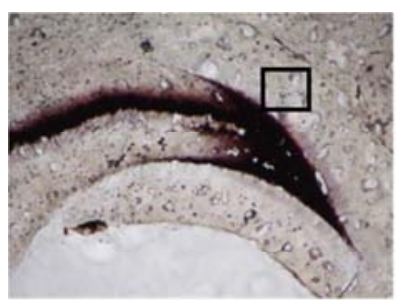

(a)

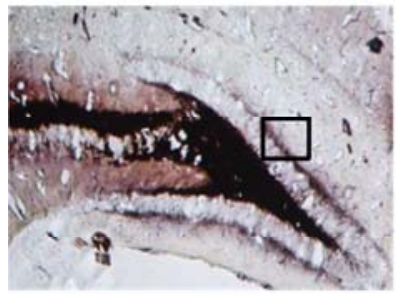

(c)

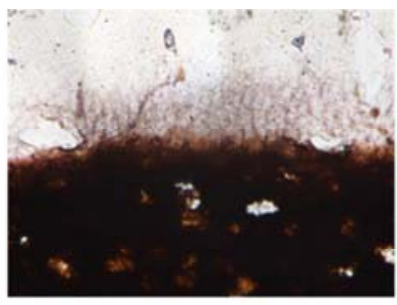

(b)

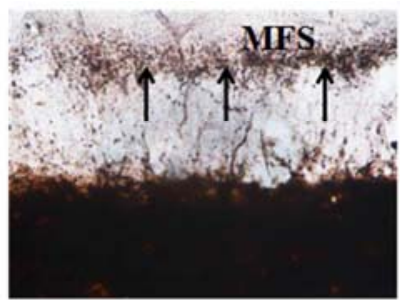

(d)

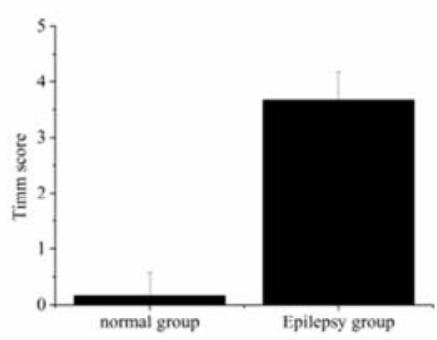

(e)

Figure 2. Timm-staining results of the hippocampi ( $A$ and $C$ ) and dentate gyrus ( $B$ and $D$ of the normal group (A and B) and the treated group (C and D). The fiber sprouting was evaluated as score as shown in Panel E.

Next, the double immunofluorescence staining experiments were performed on $\sim \mu$ m-thick brain sections. When anti 14-3-3 zeta protein primary antibody and Alexa-488 fluorescently labeled secondary antibody was used, as shown in Figure 3D, the excitation of $800 \mathrm{~nm}$ gave rise to the significant green fluorescent signals in CA1 domain of TLE. No similar signals were detected for the control group (seen in Figure 3A). It indicated that the position change of 14-3-3 zeta protein might be implicated in the development of TLE (In TLE, 14-3-3 zeta was mainly present in CA1 zone). As for p-Tau (Ser262), one could clearly observe the red fluorescence signals coming from the Alexa-569 fluoresent dye-labeled IgG that was combined to anti p-Tau (Ser262) primary antibody. Comparisons showed that p-Tau (Ser262) also experienced some spatial variations from the control group (Figure 3B) to TLE group. When Figure 3C and 3D were merged, there appeared the yellow signals in CA1 zone, indicating that 14-3-3 zeta protein and p-Tau (Ser262) were colocalized in CA1 zone. Similar phenomenon was also detected in CA3 zone of TLE mice (data not shown).

In the next work, the 3D architecture and the spatial relationship between 14-3-3 zeta and p-Tau (Ser262) were further investigated by combining CLARITY and immunofluorescent staining. Firstly, passive CALRITY strategy was conducted on 3mm-thick hippocampus blocks according to the protocol described in 2.3 section. Within one week these brain blocks become fully transparent with the removal of lipids, as demonstrated in Figure 4A and 4B. 

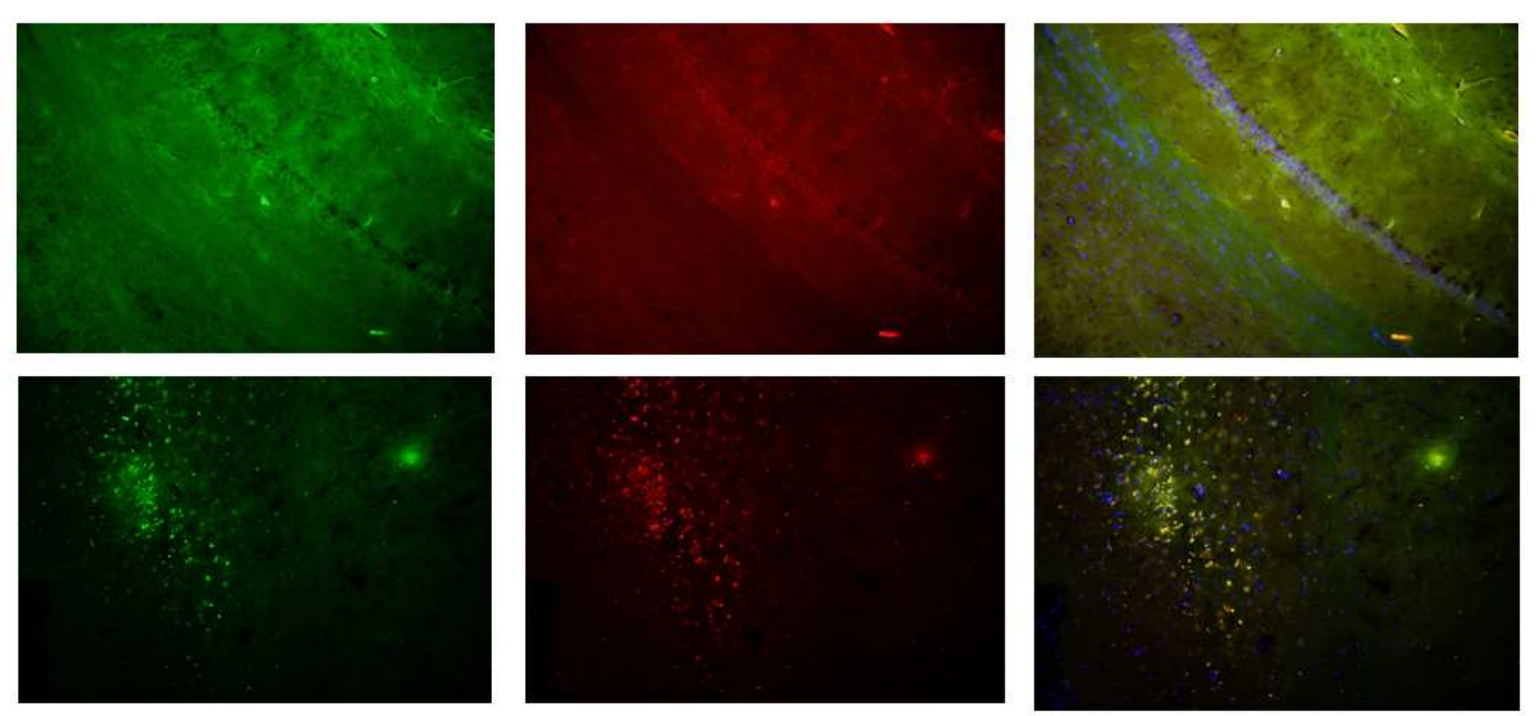

Figure 3. Immunofluorescence results of brain slices of the normal group (A-D) and the treated group (E-H). The secondary Anti-14-3-3 zeta antibody, Anti-p-262 Tau protein, and anti-MAP2 antibodies were labeled by Alexa 488, Alexa 569 and Alexa 488, respectively. DAPI was used to stain the nucleus.
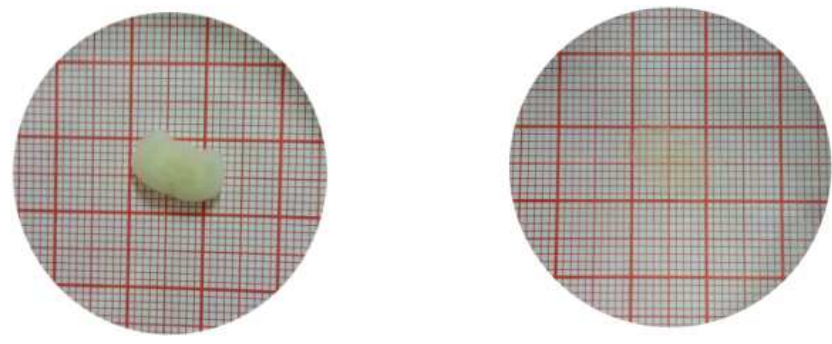

Figure 4. The $\sim 3 m m$-thick hippocampus slabs before and after the clarified treatment using passive diffusion methods.

Secondly, double immunofluorescent staining experiments were also performed on these clarified brain blocks to achieve $3 \mathrm{D}$ view of 14-3-3 zeta and p-Tau (Ser262). Figure 5 showed the reconstructed 3D fluorescence imaging of $200-\mu \mathrm{m}$-thick hippocampus region, in which the green spots and red spots represented 14-3-3 zeta and p-Tau (Ser262), respectively. It was observed that (1) p-Tau (Ser262) had diverse cluster-like structures: some were small and condensed, some were large and loose. These size variations might be due to the different aggregation degree of p-Tau (Ser262). (2) The morphology of 14-3-3 zeta was strip-shaped and heterogeneous. When compared with p-Tau (Ser262), 14-3-3 zeta protein had smaller volume than p-Tau (Ser262). (3) Different from the remarkable yellow signals merged on the $\mu \mathrm{m}$-thick brain section (Figure 3F), only weak yellow signals could be detected from the 3D resconstruction imaging (Figure 4) of the clarified mm-thick brain sections. It might be due to the volume expansion of brain tissues induced by the formation of the hydrogel matrix. Nevertheless, it still supported that there existed the spatial colocalization between 14-3-3 zeta protein and p-Tau (Ser262). This spatial relationship might provide one possibility that $14-3-3$ zeta protein might interact with p-Tau (Ser262).

It has been found that there occurred the interaction between 14-3-3 zeta and p-Tau (Ser262) in neurodegenerative disease $\mathrm{AD}$ by co-immunoprecipitation experiment. Immuno-electron microscopy results showed that as the time of incubation with 14-3-3 zeta increased, the morphology of Tau varied from the amorphous aggregation, single-stranded filaments, ribbonlike filaments to finally the paired helical filaments (PHFs). It suggested that 14-3-3 zeta protein in the association complex played a key role to regulate the structure of Tau, finally resulting in the progressive of AD. In our case, although the colocalization between 14-3-3 zeta and Tau was observed, it is unclear if 14-3-3 zeta could regulate the morphologic changes of Tau. More systematic work needs to be done to gain deeper understanding on the role of 14-3-3 zeta in such association complex.

Apart from these two proteins, MAP2 antibody was also used to response the changes of the dendritic morphology. It was observed that the dendrite changed from regular, long, silk-like shape to unordered, short, condensed-shape after the mice suffered from epilepsy (data not shown).

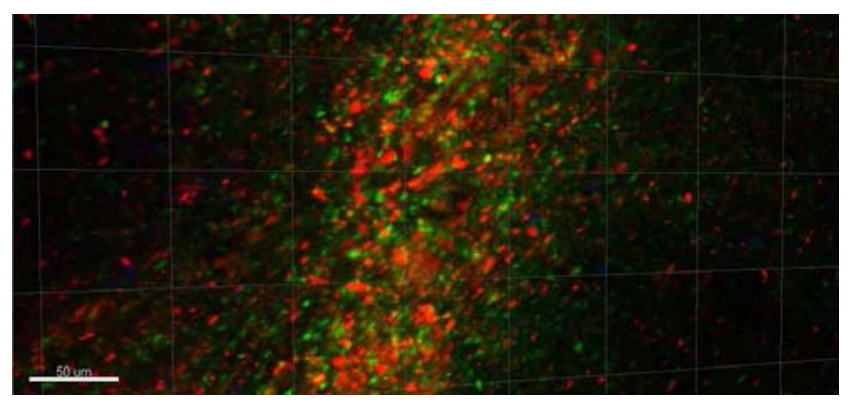

Figure 5. Immunofluorescence results of the mm-thick brain slices of the treated group. The secondary Anti-14-3-3 zeta antibody, Anti-p-262 Tau protein, were labeled by Alexa 488, Alexa 569 and Alexa 488, respectively. DAPI was used to stain the nucleus.

\section{Conclusion}

In this work we used the repeated administration of pilocarpine at 20-min intervals to produce SE with the 
mortality rate of mice $(<25 \%)$, and this model was identified using Timm-staining. Firstly, the double immunofluorescent staining on $\mu \mathrm{m}$-thick brain sections showed that different from the control group, 14-3-3 zeta and Tau colocalized in CA1 and CA3 zone. This result inferred that the position alteration of these two proteins were implicated with the progressive of TLE. Next, the mm-thick brain slices were clarified using passive diffusion method within one week. The strong fluorescence signals were observed in double immunofluorescent staining on mm-thick brain sections. It suggested that anti-14-3-3 zeta antibody and anti-Tau antibody had smoothly diffused into the porous brain tissue. Meanwhile, these antibodies exhibited high specificity aganist the target proteins under the present condition. Even though, some CLARITY conditions still need to be optimized in the further work. Additionally, the 3D reconstruction imaging revealed that the morphology of was cluster-like shape, which resembled the amorphous aggregation observed in $\mathrm{AD}$ brain. It remains unclear if this structure will experience some variations during the development of TLE. In contrast to Tau, 14-3-3 zeta protein exhibited smaller volume. More importantly, the immunofluorescent staining results showed that 14-3-3 zeta protein was colocalized with p-Tau (Ser262) in CA1 zone and CA3 zone. This spatial relationship provided one possibility that $14-3-3$ zeta protein might interact with p-Tau (Ser262), and affect the morphology of Tau. Since CLARITY can provide more direct and higher resolution 3D view than immuno-electron microscopy, in the further work, it will be necessary to apply CLARITY to explore this question by the administration of drugs or using 14-3-3 zeta-overexpressing transgenic mice to change the expression level of 14-3-3 zeta in vivo.

\section{References}

[1] Chung K, Wallace J, Kim SY, et al. Structural and molecular interrogation of intact biological systems. Nature, 2013, 497(7449): 332- 337

[2] Dodt HU, Leischner U, Schierloh A, et al. Ultramicroscopy: three-dimensional visualization of neuronal networks in the whole mouse brain. Nat Methods, 2007, 4(4): 331-336

[3] Ertürk A1, Becker K, Jährling N, et al. Three-dimensional imaging of solvent-cleared organs using 3DISCO. Nat Protoc, 2012, 7(11): 1983-1995.

[4] Hama H, Kurokawa H, Kawano H, et al. Sca/e: a chemical approach for fluorescence imaging and reconstruction of transparent mouse Brain. Nat Neurosci, 2011, 14(11): 1481-1488.

[5] Ke MT, Fujimoto S, Imai T. See DB: a simple and morphology-preserving optical clearing agent for neuronal circuit reconstruction. Nat Neurosci. 2013, 16(8): 1154-1161.

[6] Susaki EA, Tainaka K, Perrin D, Whole-brain imaging with single-cell resolution using chemical cocktails and computational analysis. Cell. 2014, 157(3): 726-739.
[7] Tomer R, Ye L, Hsueh B, et al. Advanced CLARITY for rapid and high-resolution imaging of intact tissues. Nat Protoc, 2014, 9(7): 1682-1697

[8] Lee H, Park JH, Seo I, et al. Improved application of the electrophoretic tissue clearing technology, CLARITY, to intact solid organs including brain, pancreas, liver, kidney, lung, and intestine. BMC Dev Biol., 2014,14:48. doi: 10.1186/s12861-014-0048-3

[9] Bastrup J, Larson PH. Optimized CLARITY technique detects reduced parvalbumin density in a genetic model of schizophrenia. Journal of neuroscience methods, 2017, 283: 23-32

[10] Deisseroth K. Optical and chemical discoveries recognized for impact on biology and psychiatry. EMBO Reports, 2017, 18(6): $859-860$

[11] Milgroom A, Ralston E. Clearing skeletal muscle with CLARITY for light microscopy imaging. Cell Biology International, 2016, 40(4): 478-483

[12] Muzumdar MD, Dorans KJ, Chung KM, et al. Clonal dynamics following p53 loss of heterozygosity in Kras-driven cancers. Nature Communications, 2016, 7: 12685.

[13] Ding Y, Lee J, Ma J, et al. Light-sheet fluorescence imaging to localize cardiac lineage and protein distribution. Scientific Reports, 2017, 7: 42209. doi: 10.1038/srep42209

[14] Leuze C, Aswendt M, Ferenczi E, et al. The separate effects of lipids and proteins on brain MRI contrast revealed through tissue clearing. Neuro Image, 2017, 156: 412-422

[15] Syed AM, Wilhelm S, Glancy DR. Three-dimensional optical mapping of nanoparticle distribution in intact tissues. ACS Nano, 2016, 10(5): 5468-5478

[16] Spence RD, Kurth F, Itoh N, et al. Bringing CLARITY to gray matter atrophy. Neuroimage., 2014, 101: 625-632.

[17] Ando K, Laborde Q, Lazar A, et al. Inside Alzheimer brain with CLARITY: senile plaques, neurofibrillary tangles and axons in 3-D. Acta Neuropathol., 2014, 28(3): 457-459.

[18] Liu AK, Hurry ME, Ng OT, et al. Bringing CLARITY to the human brain: visualization of Lewy pathology in three dimensions [J]. Neuropathol Appl Neurobiol, 2016, 42(6): 573-587.

[19] Umahara T, Uchihara T, Tsuchiya K, et al. 14-3-3 proteins and zeta isoform containing neurofibrillary tangles in patients with Alzheimer's disease. Acta Neuropathol. 2004, 108(4):279-286. b) Sluchanko NN, Seit-Nebi AS, Gusev NB. Effect of phosphorylation on interaction of human tau protein with 14-3-3zeta. Biochem Biophys Res Commun. 2009, 379(4): 990-994.

[20] Qureshi HY, Li T, MacDonald R, et al. Interaction of 14-3-3 zeta with microtubule-associated protein tau within Alzheimer's disease neurofibrillary tangles. Biochemistry. 2013, 52(37):6445-6455.

[21] Glien M, Brandt C, Potschka H, et al. Repeated low-dose treatment of rats with pilocarpine: low mortality but high proportion of rats developing epilepsy [J]. Epilepsy Res, 2001,46(2): 111-119. 